\title{
Developing Interactive Learning for Continuous Glucose Monitoring
}

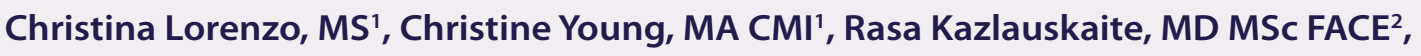

Donna Hughes, MA' ${ }^{1}$, Leah Lebowicz, MS CMI'

${ }^{1}$ University of Illinois at Chicago, ${ }^{2}$ Rush University Medical Center

\section{Abstract}

This research project investigates the development of an interactive visual learning tool for patients utilizing continuous glucose monitoring. A beta product of this interactive learning tool was developed to run real-life scenarios that visualize how meal choices and medication affect glucose trajectories in both healthy patients and patients with type 2 diabetes. This learning tool is intended to be used by the clinician to engage the patient in an educational discussion about continuous glucose monitoring. The tool may enhance a patient's understanding of graphic glucose trajectories so that they may discover how their body reacts to different lifestyle choices and learn to make better real-time decisions with their own CGM data. The effectiveness of this beta product was evaluated by 17 health professionals.

\section{Introduction}

The most recent National Diabetes Statistics Report estimates that 29.1 million people in the United States have diabetes ${ }^{2}$. Of these 29.1 million people with diabetes, as many as 90 to 95 percent of them have type 2 diabetes ${ }^{1}$. There is an enormous need to develop new methods for managing diabetes and educating these current and potential future patients.

The newest technology in diabetes management is the continuous glucose monitoring (CGM) system. The CGM provides glucose readings every five minutes and allows a patient to see their real-time graphic glucose trajectories over a 24-hour period ${ }^{3}$. The CGM is comprised of a sensor, transmitter, and receiver. Glucose measurements are taken from the subcutaneous interstitial fluid by the sensor, relayed by the transmitter, and displayed in a readable graph format and stored on the mobile receiver ${ }^{4}$. Since this technology is relatively new, there are no associated patient education materials that inform patients about glucose trajectories or how dietary choices influence change in these glucose trajectory graphs.

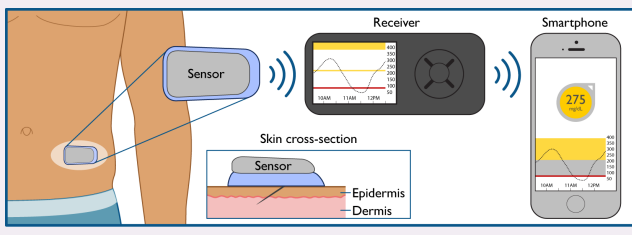

Figure 1. Data flows between the CGM sensor, receiver, and smartphone.

\section{Methods of Production}

\section{Preparation Phase}

- Background information

collected on patient

demographics, diabetes

education, and CGM

education

- Learning objectives defined

with the content expert's

oversight

- De-identified CGM data

sets compiled to create

glucose trajectory curves

\section{Prototype}

Stills from the interactive learning tool showing the start screen, four scenarios and conclusion.
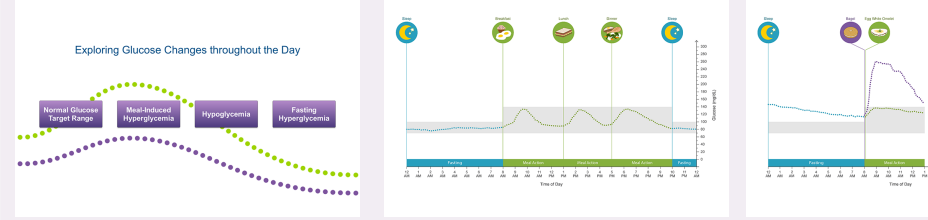

Figure 2. Start Screen

Figure 3 . Normal Glucose Target Range
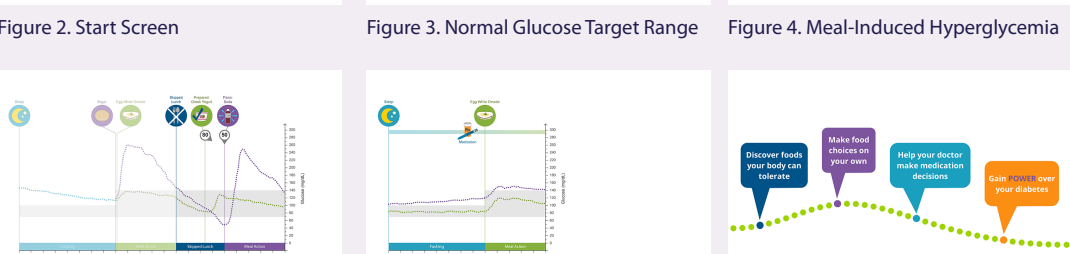

Figure 5. Hypoglycemi
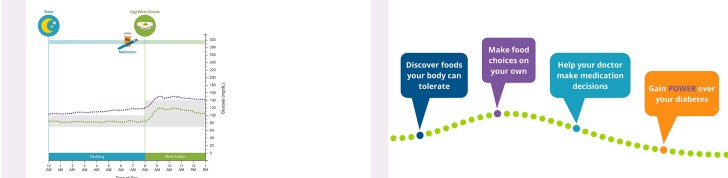

Figure 6. Fasting Hyperglycemia

Figure 7. Conclusion

\section{Interview Procedures}

The study interviewed 17 health professionals who directly interact with patients who have type 2 diabetes. The audio-recorded interviews included a questionnaire and glucose graphicacy test. Scenario questions collected feedback on the effectiveness of the visuals, complexity of the material the desire of use, and areas for improvement. Summary questions collected feedback on the appropriate duration, ideal setting for use, plausibility of patients deciding to wear CGM after viewing the tool, and areas for improvement. The study also collected feedback on how the graphicacy test was administered and the effectiveness of the test in the context of CGM.

\section{Results}

A visual interactive learning tool can be developed to explain how eating behaviors affect the dynamic shape of the glucose trajectory graph created by the continuous glucose monitor.

- Animated visuals, including icons, labels, trajectory curves, and graphs, are an effective method for communicating the topics of normal glucose target range, meal-induced hyperglycemia, hypoglycemia, and fasting hyperglycemia.

- The tool is at an appropriate learning level and length for its targeted patient population as established by health professionals.

- Health professionals indicated that they would incorporate this interactive learning tool into their discussion with patients about CGM at the clinic and would offer it as a standalone tool to patients at home.

- Health professionals indicated that it is highly plausible that patients may be more inclined to wear CGM after the presentation of this tool.

\section{Discussion}

To the author's knowledge, this interactive learning tool is the first educational tool of its kind to support an understanding of graphic data presented to patients with type 2 diabetes utilizing CGM. This study has shown promising results supporting this tool's use as an effective method for helping patients understand how lifestyle choices affect their glucose levels. The next step in this research will be to test the refined prototype on patients with diabetes utilizing CGM. It is planned that this learning tool will be available in the clinic and online.

\section{References}

American Diabetes Association. (2017). Classification an Diagnosis of Diabetes. Diabetes Care, 40(Supplement 1), S11-S24. Diagnosis of Diabetes. D
doi: $10.2337 /$ dc $17-5005$

2. Centers for Disease Control and Prevention. (2014). National Diabetes Statistics Report: Estimates of Diabetes and Its Burden in 3. Dexcom G5 Mobile CGM System. (2016). Retrieved July 11, 2016, from http://www.dexcom.com/g5-mobile-cgm

4. Wahowiak, L. (2017, March/April). Everything You Need to Know About Continuous Glucose Monitors. Diabetes Forecast. Retrieved from http://www.diabetesforecast.org/2017/mar-apr/continuous-glucose-monitors.html 